\title{
Role of Multistep Processes in Heavy-Ion Inner-Shell Excitations
}

\author{
J. Reinhardt, B. Müller, and W. Greiner \\ Institut für Theoretische Physik der Johann Wolfgang Goethe Universität, 6000 Frankfurt am Main, Germany
}

and

\author{
G. Soff \\ Gesellschaft für Schwerionenforschung, 6100 Darmstadt, Germany
}

(Received 9 July 1979)

\begin{abstract}
This Letter discusses inner-shell excitation in collisions of very heavy ions $\left(Z_{1}+Z_{2}\right.$ $z 140$ ) in the framework of the quasimolecular model. The importance of multistep excitations and of coupling between continuum states is demonstrated. The 1so vacancy probabilities resulting from coupled-channels calculations exceed perturbation theory by a factor 3-5, thus giving good agr eement with recent experimental results.
\end{abstract}

The excitation of inner shells in collisions of heavy ions at energies up to the nuclear Coulomb barrier is best described in the quasimolecular model. Here one starts from the solutions of the stationary two-center problem and treats the excitations as being caused by the explicit time dependence of the electronic Hamiltonian. Calculations along these lines ${ }^{1}$ have predicted large vacancy-formation rates for superheavy systems. These results have subsequently been confirmed in several experiments. ${ }^{2-6}$ Whereas the measured impact-parameter (bombarding energy) and charge dependence was found in good agreement with the predictions of first-order perturbation theory (in powers of $\theta / \partial t$ ), there remained an unexplained discrepancy in absolute magnitude: Experimental data lay above theory by a factor of $3-5$.

In this Letter we point out that it is important to go beyond perturbation theory ${ }^{7}$ to obtain quantitative agreement. We demonstrate the importance of multistep processes, where an electron is excited via various intermediate states, by the first coupled-channels calculations performed for collision systems with relativistic binding. In the following, we will first discuss the treatment of excitation of a single electron and then show that the effect of the Pauli principle can be easily implemented. In the second part of the Letter, numerical results will be presented.

We start from the complete set of stationary solutions of the two-center Dirac equation

$$
H_{\mathrm{TCD}}(R) \varphi_{i}(R)=E_{i}(R) \varphi_{i}(R)
$$

which is parametrized by the internuclear distance $R$. The time dependence $R(t)$ is prescribed by the nuclear trajectory. To solve the time- dependent Dirac equation

$$
H_{\mathrm{TCD}}[\overrightarrow{\mathrm{R}}(t)] \varphi_{i}^{(+)}(t)=i(\partial / \partial t) \varphi_{i}^{(+)}(t)
$$

with the initial condition $\varphi_{i}^{(+)}(-\infty)=\varphi_{i}$ we expand the wave function

$$
\begin{aligned}
\dot{a}_{i j}(t)=-\sum_{k} a_{i k}(t)\left\langle\varphi_{j}|\partial / \partial t| \varphi_{k}\right\rangle \\
\times \exp \left[-i\left(\chi_{k}-\chi_{j}\right)\right]
\end{aligned}
$$

with the phase $\chi_{j}=\int_{t_{0}}^{t} d t^{\prime} E_{j}\left(R\left(t^{\prime}\right)\right)$. Projection on a particular basis state yields the coupled differential equations

$$
\begin{aligned}
\dot{a}_{i j}(t)=-\sum_{k} a_{i k}(t)\left\langle\varphi_{j}|\partial / \partial t| \varphi_{k}\right\rangle \\
\times \exp \left[-i\left(\chi_{k}-\chi_{j}\right)\right]
\end{aligned}
$$

subject to the initial condition $a_{i j}(-\infty)=\delta_{i j}$. Here and in the following the sum implies an integral over the continuous spectrum. The squared amplitude $\left|a_{i j}\right|^{2}$ denotes the probability that the electron has been excited from state $i$ to state $j$ during the course of the collision. The amplitudes $a_{i j}$ completely describe a collision where a single electron is present. Normally, however, the projectile ion is only partially stripped and the target atom is neutral so that several bound states are occupied.

If pair creation is to be included in the description, one even has, in principle, to deal with infinitely many particles. Knowledge of the singleparticle amplitudes $a_{i j}$, however, is still sufficient if one neglects the two-body part of the electron-electron interaction in the Hamiltonian $H_{\mathrm{TCD}}$. The Pauli principle does not lead to complications since the "dynamic" wave functions $\varphi_{i}{ }^{(+)}(t)$ which contain the time development of single-electron states are mutually orthogonal at every instant of time because of the unitarity of the time development operator. The set $\varphi_{i}^{(+)}$, 
therefore, can be used as a basis for describing the many-particle problem. ${ }^{8}$

We can expand the (time-dependent) field operator in the basis $\varphi_{i}^{(+)}$so that

$$
\hat{\psi}=\sum_{i<F} \hat{d}_{i}^{\dagger} \varphi_{i}^{(+)}+\sum_{i>F} \hat{b}_{i} \varphi_{i}^{(+)}
$$

where $\hat{b}_{i}, \hat{b}_{i}^{\dagger}$ and $\hat{d}_{i}, \hat{d}_{i}^{\dagger}$ are particle and hole operators, respectively, which satisfy the usual anticommutation relations. The operators $\hat{b}_{i}$ and $\hat{d}_{i}$ are defined such that they annihilate the (Heisenberg) state $|F\rangle: \hat{b}_{i}|F\rangle=0$ for $i>F$ and $\hat{d}_{i}|F\rangle=0$ for $i<F$. To determine the asymptotically observed particle excitation we use the alternative expansion

$$
\hat{\psi}=\sum_{i<F} \hat{\tilde{d}}_{i}^{\dagger} \varphi_{i}^{(-)}+\sum_{i>F} \hat{\tilde{b}}_{i} \varphi_{i}^{(-)}
$$

where $\varphi_{i}^{(-)}$are the solutions to Eq. (2) satisfying the condition $\varphi_{i}^{(-)}(+\infty)=\varphi_{i}$. Equating (5) with (6) leads to a canonical transformation between the two sets of operators $\hat{b}_{i}, \hat{d}_{i}$ and $\hat{\tilde{b}}_{i}, \hat{\widetilde{d}}_{i}$. The number of particles excited into an initially vacant level $\varphi_{i}$ (irrespective of the occupation of the other states) then reads

$$
N_{i}=\left\langle F\left|\hat{\tilde{b}}_{i}^{\dagger} \hat{\tilde{b}}_{i}\right| F\right\rangle=\sum_{k<F}\left|a_{k i}\right|^{2}, \quad i>F,
$$

and the number of holes in an initially occupied state $\varphi_{j}$ is

$$
N_{j}=\left\langle F\left|\hat{\tilde{d}}_{i}^{\dagger} \hat{\tilde{d}}_{i}\right| F\right\rangle=\sum_{k>F}\left|a_{k j}\right|^{2}, \quad j<F .
$$

Thus the particle (hole) excitation probability in a particular state is simply given by an incoherent sum of single-electron transition probabilities as shown by Reading (Ref。8)。 The result is influenced by the number of electrons present solely via the position of the Fermi surface $F$. The number of correlated pairs, however, is not just the product of single excitation numbers. For particle-hole pairs $(i>F, j<F)$ we find

$$
\begin{aligned}
N_{i, j} & =\left\langle F\left|\hat{\tilde{b}}_{i}^{\dagger} \hat{\tilde{b}}_{i} \hat{\tilde{d}}_{j}^{\dagger} \hat{\tilde{d}}_{j}\right| F\right\rangle \\
& =N_{i} N_{j}-\sum_{k<F} \sum_{l>F} a_{k i} * a_{k j} a_{l i} * a_{l j} \\
& =N_{i} N_{j}+\left|\sum_{k<F} a_{k i} * a_{k j}\right|^{2} .
\end{aligned}
$$

This formula, which was also derived independently by Ford and Reading, 9,10 should be applied to analyze experiments like those presently performed for coincidences between electrons and characteristic $\mathrm{x}$ rays. Equation (9) holds also for particle-particle or hole-hole correlations if the sign of the second term is changed. If the calculation involves channels which are treated incoherently, e.g., spin-up and spin-down states when neglecting rotational coupling and magnetic effects, Eq. (9) has to be supplemented by incoherent terms to account for random coincidences. The mentioned case of spin degeneracy leads to an enhancement by more than a factor of 2 , namely

$$
N_{i, j}=2 N_{i, j}{ }^{0}+2 N_{i}^{0} N_{j}^{0},
$$

where $N_{i}{ }^{0}, N_{j}^{0}$, and $N_{i, j}{ }^{0}$ are calculated from Eqs. (7)-(9) for one spin state and $N_{i, j}$ is summed over all combinations.

Having established the relevance of the singleparticle amplitudes, we must turn to the solution of the coupled differential Eqs. (4). For any practical use the basis has to be restricted to a finite and not too large set. In particular, the continuum must be discretized into finite segments. This is most simply achieved by replacing the integral $\int_{m c}^{\infty} d E f(E)$ by a finite sum $\sum_{i} \Delta E_{i} f\left(E_{i}\right)$. Since the matrix elements vary smoothly with energy, the allowed spacing $\Delta E_{i}$ is limited by the oscillations of the phase $\chi_{E}(t)$ : $\Delta x=(\Delta E)(\Delta t) / \hbar \ll \pi$. If we assume a mean excitation region of $\Delta R=300 \mathrm{fm}$ and collision velocity $v / c \approx 0.1$, this condition gives roughly $\Delta E \ll 0.4 m c^{2}$. Numerical calculations are feasible down to $\Delta E$ $\approx 0.1 m c^{2}$ at low electron kinetic energies. The upper energy limit for states to be included is determined by the Fourier spectrum of the collision and by the energy dependence of the coupling matrix elements. We have tested the latter by applying the completeness relation and checking the identity

$$
\sum_{k}|\langle k|\partial / \partial R| 1 s\rangle|^{2}=\left\langle 1 s\left|\partial^{2} / \partial R^{2}\right| 1 s\right\rangle .
$$

We found that it is satisfied sufficiently if states up to $2 \mathrm{MeV}$ kinetic energy are included. About half of the coupling strength goes into the continuum.

The coupling matrix elements were obtained in monopole approximation, ${ }^{11}$ which was proven to be remarkably good in very heavy collision systems. Only $n s \sigma$ states are included in the present calculation. Coupling to other states, in particular rotational coupling, was found to be weak in high- $Z$ near-symmetric systems. For more details on the basis used, see Soff etal. ${ }^{12}$ All radial matrix elements were extrapolated to distances $R>200 \mathrm{fm}$ by a power law and cut of smoothly with a Gaussian factor at $R>1500 \mathrm{fm}$ to simulate the effect of translation factors. The integration 


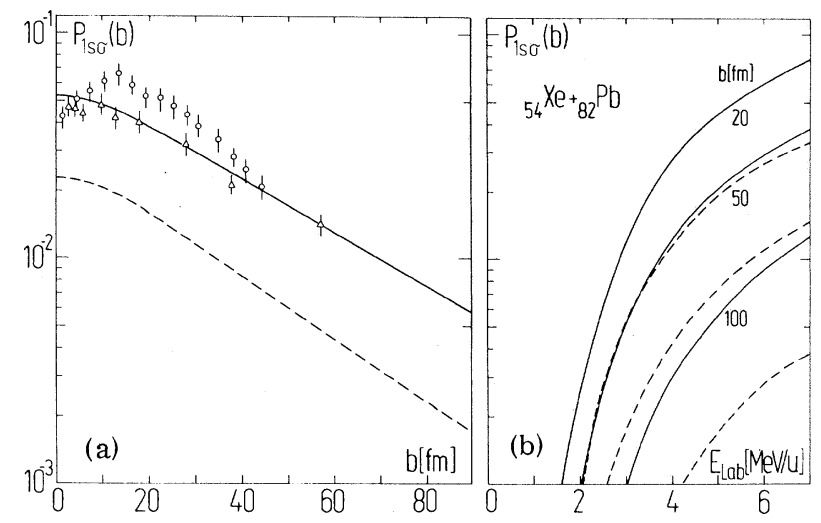

FIG. 1. (a) Probability for $1 s \sigma$ excitation as a function of impact parameter $b$ for $\mathrm{Xe}-\mathrm{Pb}$ collisions at $4.6 \mathrm{MeV} /$ $N$ laboratory energy. Full line, coupled-channels result assuming a Fermi level above $F=3 s \sigma$; dashed line, perturbation theory. Also shown are experimental results of Greenberg et al. (Ref. 3) (circles) and Anholt et al. (Ref. 14) (triangles). (b) Energy dependence of $P_{1 s \sigma}(b)$ at various fixed impact parameters.

was performed along Rutherford trajectories up to $R=3000 \mathrm{fm}$ with use of a standard predictorcorrector Hamming routine.

The most remarkable new result of the coupledchannels treatment is an increase of vacancyformation rates by up to a factor of 5 due to multistep excitations compared to previous perturbative calculations, ${ }^{1,13}$ the effect being largest for very heavy systems. To demonstrate the influence of excitation via intermediate states the Table I shows the $1 s \sigma$ hole probability (normalized to 2 because of spin degeneracy) for $\mathrm{Pb}-\mathrm{Pb}$ collision at $b=0$ and $2 a=20 \mathrm{fm}\left(E_{\mathrm{lab}} / A=4.65 \mathrm{MeV} /\right.$ $N)$. The results of several calculations including a varying number of $n s \sigma$ bound-state channels

TABLE I. $\Re$ designates the highest bound state included; $F$ is the last occupied state. Thus the left column $(F=\Re)$ includes excitations to the continuum only while in the median (right) column the Fermi surface was taken above the $3 s \sigma(1 s \sigma)$ state. All calculations include 16 continuum states. Doubling of this number results in a less than $10 \%$ change of the excitation rates.

\begin{tabular}{cccc}
\hline$\Re$ & $F=\Re$ & $F=3 s \sigma$ & $F=1 s \sigma$ \\
\hline $1 s \sigma$ & $2.96 \%$ & $\ldots$ & $\ldots$ \\
$2 s \sigma$ & $4.80 \%$ & $\ldots$ & $19.92 \%$ \\
$3 s \sigma$ & $6.84 \%$ & $\ldots$ & $21.40 \%$ \\
$4 s \sigma$ & $8.08 \%$ & $9.24 \%$ & $22.12 \%$ \\
$6 s \sigma$ & $9.06 \%$ & $11.00 \%$ & $22.56 \%$ \\
$8 s \sigma$ & $9.42 \%$ & $11.34 \%$ & $22.68 \%$ \\
\hline \hline
\end{tabular}

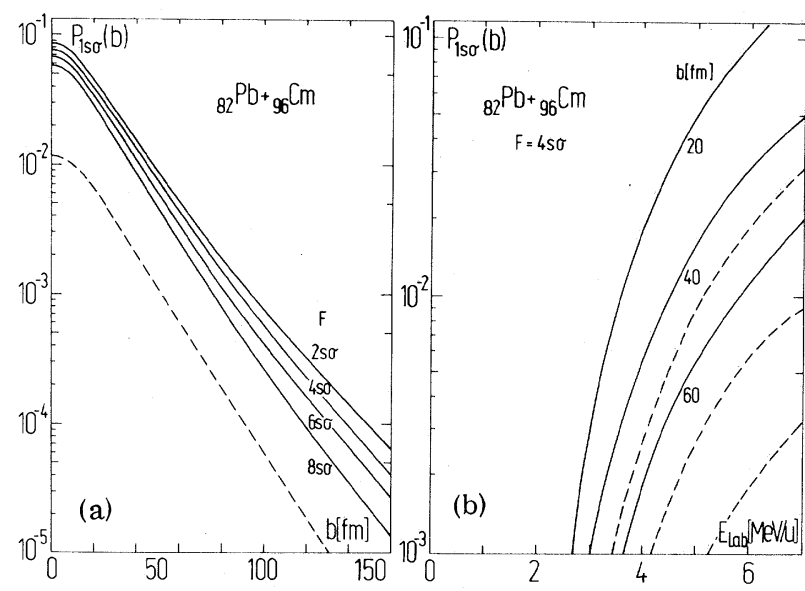

FIG. 2. Same as Fig. 1 for $\mathrm{Pb}-\mathrm{Cm}$ collisions at 4.7 $\mathrm{MeV} / N$ laboratory energy. The various curves in (a) belong to different positions of the assumed Fermi level $F$ as indicated in the figure.

are given.

The coupling between continuum states mainly leads to a shift of the spectrum: The emitted electrons are rescattered to higher energy. The inclusion of intermediate bound states increases the ionization by supplying mainly low-energy electrons so that the net effect of multiple excitations is a rise of the electron spectrum without change of shape.

Fig.1(a) shows the impact-parameter dependence of $K$-vacancy production for $\mathrm{Xe}-\mathrm{Pb}$ collisions at $E / A=4.6 \mathrm{MeV} / N$ calculated in perturbation theory (dashed line) and in the coupledchannels approach (full lines) for the (last occupied) Fermi level $F=3 s \sigma(\Re=8 s \sigma$ in all calculations). The multistep excitation is seen to lead to an overall increase of $\boldsymbol{P}(b)$. Also shown are recent experimental results obtained by two groups in Darmstadt and Stanford., ${ }^{2,14}$ Theory is in remarkable agreement with these data and also with measurements in other systems like $\mathrm{Xe}-\mathrm{Au}$ (Ref。3). Similar agreement is found for the dependence on bombarding energy ${ }_{9}^{15}$ which is shown in Fig. 1(b)。 Our theory, however, does not show a peak at small impact parameter. This special feature was observed in one of the experiments and was tentatively explained in the Briggs model ${ }^{16}$ with use of perturbation theory in the united-atom basis.

Fig. 2 gives the predicted $1 s \sigma$ vacancy probability for the very heavy system $\mathrm{Pb}-\mathrm{Cm}\left(Z_{1}+Z_{2}\right.$ $=178$ ) for various positions of the Fermi surface. Note, in particular, the strong impact-param- 
eter dependence and the steep increase with bombarding energy.

In summary, we have demonstrated the importance of multistep processes in the quasimolecular description of heavy-ion inner-shell excitations. The nonadiabacity of the electronic motion is the origin of violent coupling between neighboring states which have the effect of increasing the excitation rates. The number of vacancies depends monotonically [Eq. (8)] on the number of initially occupied states. To obtain accurate results for vacancy formation (and thus also for positron production) the initial distribution of electrons (i.e., the position of the Fermi surface) must be known. The characteristics of the excitation process are changed slightly by multistep excitation, leading to a weaker dependence on impact parameter and energy. Detailed studies of the impact-parameter, energy, and charge dependence of inner-shell excitation as a function of the binding energies and coupling strengths entering the coupled-channels calculations will be necessary for the envisaged spectroscopy of superheavy quasimolecules.

This work was supported by the Bundesministerium für Forschung und Technologie and by the Gesellschaft für Schwerionenforschung.

${ }^{1}$ W. Betz, G. Soff, B. Müller, and W. Greiner, Phys.
Rev. Lett. 37, 1046 (1976).

${ }^{2}$ J. S. Greenberg, H. Bokemeyer, H. Emling,

E. Grosse, D. Schwalm, and F. Bosch, Phys. Rev. Lett. 39, 1404 (1977).

${ }^{3}$ D. Liesen, P. Armbruster, H.-H. Behnke, and S. Hagmann, Z. Phys. A 288, 417 (1978).

${ }^{4} \mathrm{~J}$. R. Macdonald, P. Armbruster, H. H. Behncke, F. Folkmann, S. Hagmann, D. Liesen, P. H. Mokler, and A. Warczak, Z. Phys. A 284, 57 (1978).

${ }^{5}$ H.-H. Behncke, D. Liesen, S. Hagmann, P. H. Mokler, and P. Armbruster, Z. Phys. A 288, 35 (1978).

${ }^{6}$ R. Anholt and W. E. Meyerhof, Phys. Lett. $64 \mathrm{~A}, 381$ (1978).

${ }^{7}$ T. H. Rihan, B. Müller, and W. Greiner, J. Phys. B 12, 761 (1979).

${ }^{8}$ A. L. Reading, Phys. Rev. A $\underline{8}, 3262$ (1973).

${ }^{9} \mathrm{~J}$. F. Ford and A. L. Reading, to be published.

${ }^{10} \mathrm{~J}$. Reinhardt, B. Muller, and W. Greiner, in Proceedings of the Intermational Workshop on Coherence and Correlation in Atomic Collisions, edited by H. Kleinpoppen and J. F. Williams (Plenum, New York, 1979).

${ }^{11}$ G. Soff, W. Betz, J. Reinhardt, and J. Rafelski, Phys. Scr. 17, 417 (1978).

${ }^{12}$ G. Soff, W. Greiner, W. Betz, and B. Müller, Phys. Rev. A 20, 169 (1979).

${ }^{13}$ G. Soff, W. Betz, B. Müller, W. Greiner, and E. Merzbacher, Phys.Lett. 65A, 19 (1978); B. Müller, G. Soff, W. Greiner, and V. Ceausescu, Z. Phys. A 285, 27 (1978).

${ }^{14}$ R. Anholt, W. E. Meyerhof and Ch. Stoller, Z. Phys. A 291,1287 (1979).

${ }^{15} \mathrm{D}$. Liesen, private communication.

${ }^{16}$ P. A. Amundsen, J. Phys. B 11 , L737 (1978).

\section{Observations on the $v=0 \rightarrow v=5$ Rotational-Vibrational Band of HD \\ F. W. Dalby ${ }^{(a)}$ and J. Vigué \\ Laboratoire de Spectroscopie Hertzienne de l'Ecole Normale Supérieure, Université Pierre et Marie Curie, 75230 Paris Cédex 05, France, and Department of Physics, University of British Columbia, Vancouver, British Columbia V6T 1W5, Canada \\ (Received 21 August 1979)}

The $v=0 \rightarrow v=5$ rotational-vibrational spectrum of free HD has been observed by means of photoacoustic spectroscopy. The electric dipole matrix elements are found to depend significantly upon the rotational quantum number. The dependence of the frequency of the $R(1)$ line upon density has been determined.

Electric dipole vibrational spectra of free $\mathrm{H}_{2}$ molecules are rigorously forbidden by symmetry. For HD, electric-dipole spectra are permitted by symmetry but are very weak, being allowed only because of nonadiabatic effects. They are forbidden within the Born-Oppenheimer approximation. The first observation of a HD dipole vibrational spectrum was made by Herzberg. ${ }^{1}$
Trefler and Gush ${ }^{2}$ have studied the pure rotational spectrum. McKellar, Goetz, and Ramsay ${ }^{3}$ have reported wavelength and intensity measurements of the stronger lines in the $3-0,4-0,5-0$, and $6-0 \mathrm{HD}$ bands (we denote $v=0 \rightarrow v_{f} \neq 0$ by $v_{f}-0$ hereafter) and surveyed earlier work. The spectral properties of HD are of astronomical importance as the vibrational spectrum of HD has been 